\title{
Hughes-Stovin syndrome complicated with fatal pneumothorax in Behçet's disease: simple bad luck or is there an etiopathogenic link?
}

\begin{abstract}
Pulmonary involvement in Behçet's disease is not usual, and the occurrence of spontaneous pneumothorax is evena rarer complication. We report the case of a secondary spontaneous pneumothorax occurring in a patient aged 21 diagnosed of Hughes-Stovin syndrome treated with colchicine, corticosteroids and cyclophosphamide. The patient experienced chest pain with acute dyspnea. The examination found an increase in the sonority during percussion of the thorax with a decrease in respiratory sounds in auscultation. Chest X-ray and thoracic CT scan showed the presence of a left medium-sized pneumothorax that extended rapidly and became bilateral. The patient was transferred to a resuscitation unit undergoing a chest drainage. The course was marked by severe respiratory distress requiring intubation. The pneumothorax was probably caused by the rupture of pneumatocele in the pleural cavity. Several factors have contributed to pneumatocele formation, including pulmonary pneumonitis and mechanical ventilation in a lung already weakened by the inflammatory process of Behçet's vasculitis. The patient had died with an unexplained etiology.
\end{abstract}

Volume 13 Issue 2 - 2020

\author{
Mayssem Jridi,' Imène Rachdi,' Fatma \\ Daoud,' Mehdi Somai, 'Wafa Garbouj, ${ }^{2}$ Hana \\ Zoubeidi,' Wided Hizem,' Zohra Aydi,' \\ Besma Ben Dhaou,' Fatma Boussema' \\ 'Department of Internal Medicine, University of Tunis El Manar \\ Tunisie \\ 2Department of Internal Medicine, Mohamed Tahar Maamouri \\ Hospital,Tunisia
}

Correspondence: Imène Rachdi, Department of Internal Medicine-Habib Thameur Hospital- Faculty of Medicine of Tunis, University of Tunis El Manar,Tunisia, Email rachdi.imenel4@gmail.com

Received: February 24, 2020 | Published: April I5, 2020

Keywords: behçet's disease, hughes-stovin syndrome, pneumothorax

\section{Introduction}

The pulmonary manifestations during Behçet's disease (BD) are essentially of the vascular type: pulmonary embolism and pulmonary arterial aneurysms. ${ }^{1}$ The occurrence of spontaneous pneumothorax is not a usual complication during BD. We report the case of a secondary spontaneous pneumothorax occurring in a patient diagnosed of Hughes-Stovin syndrome.

\section{Observation}

A 21-year-old man was followed for six months in the internal medicine department for Hughes-Stovin syndrome. He presented with pulmonary arterial aneurysms (Figure 1) thrombosis of the hepatic and inferior vena cava veins and recurrent bipolar aphthosis. The patient was treated with colchicine mg per day, oral corticosteroid $60 \mathrm{mg}$ per day and monthly boli of cyclophosphamide. The evolution was marked by the occurrence of a state of epilepticus complicated by inhalation pneumopathy due to Staphylococcus Aureus requiring intubation and mechanical ventilation for eight days in intensive care unit. Status epilepticus could not be explained by metabolic, toxic, infectious or iatrogenic causes. There was no concept of trauma and the brain scanner was without abnormalities. Magnetic resonance brain imaging with gadolinium injection showed no parenchymal or vascular lesions. The electroencephalogram showed a non-abnormal pattern. An improvement in the neurological state with no seizure recurrence was obtained under anti-epileptic treatment. The pulmonary examination showed clinical improvement and radiological cleansing. Two weeks later, the patient experienced chest pain with acute dyspnea. The examination found an increase in the sonorityduring percussion of the thorax with a decrease in respiratory sounds in auscultation. Chest X-ray and thoracic computed tomography scan showed the presence of a left medium-sized pneumothorax (Figure $2 \mathrm{~A}$ ), associated with fusiform aneurysmal dilatationof the apical branch of the Lingula (Figure 2B), and posterolateral atelectasis of the left lower lobe containing pneumatocele blebs. The pneumothorax extended rapidly and became bilateral. The patient was transferred to a resuscitation unit undergoing a chest drainage. The course was marked by severe respiratory distress requiring intubation. The patient had died with an unexplained etiology.

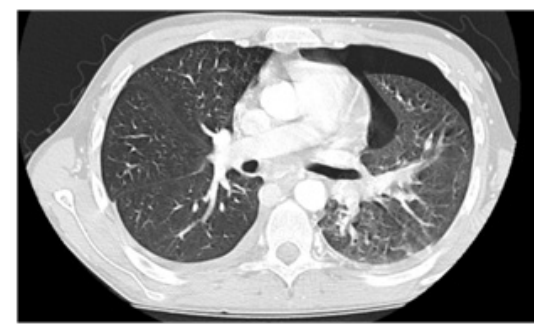

Figure I Pulmonary aneurysmsat thoracic computed tomography scan.

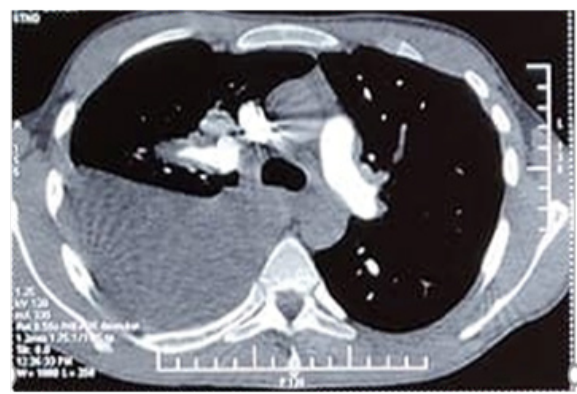

(A) 


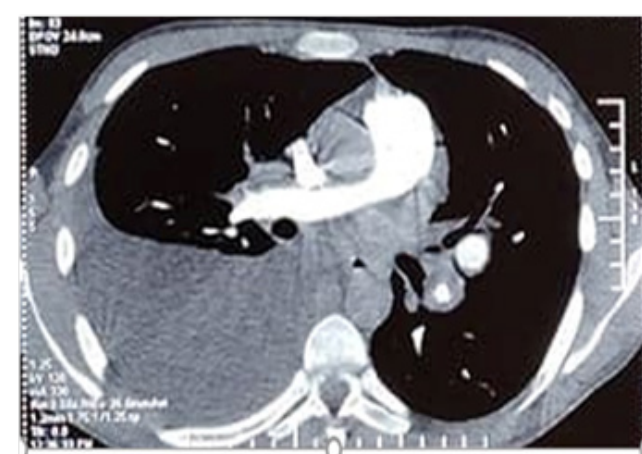

(B)

Figure 2 Left pneumothorax (A) and pulmonary anevrysms (B) at thoracic computed tomography scan.

\section{Discussion}

Spontaneous pneumothorax during BDis a non-habitual manifestation. To our knowledge, it has only rarely been reported in the literature. ${ }^{2,3}$ The hypothesis of rupture of a pneumatocele in the pleural cavity seems plausible. Several factors have contributed to pneumatocele formation, including pulmonary pneumonitis and mechanical ventilation in a lung already weakened by the inflammatory process of Behçet's vasculitis. On the other hand, the patient was treated with colchicine for 6 months. This treatment suggested as being a risk factor for pneumothorax. ${ }^{2}$ Our patient passed away two weeks after he's been admitted to resuscitation unit.Mortality rates seem to be important in secondary spontaneous pneumothorax compared to primary spontaneous pneumothorax. ${ }^{4}$

\section{Conclusion}

This case describes an association of multiple vascular and parenchymal pulmonary manifestations occurring during Behçet's disease, including secondary spontaneous pneumothorax.

\section{Acknowledgments}

None.

\section{Conflicts of interest}

The authors declare there are no conflicts of interest related to the article.

\section{Funding}

None.

\section{References}

1. Erkan F, Gül A, Tasali E. Pulmonary Involvement in Behçet's Disease. Thorax. 2001;56:572-578.

2. Gülyüz ÖC, Arslan S. An unusual complication of Behcet disease: spontaneous bilateral pneumothorax. Am J Emerg Med. 2016;34(7):1320.

3. Abadoğlu O, Osma E, Uçan ES, et al. Behcet's disease with pulmonary involvement, superiorvena cava syndrome, chyloptysis and chylous ascites. Respir Med. 1996;90(7):429-431.

4. Tanaka F, Itoh M, Esaki H, et al. Secondary spontaneous pneumothorax. Ann Thorac Surg. 1993;55:372-376. 